\title{
Potential role of chimeric genes in pathway-related gene co-expression modules
}

\author{
Piaopiao Li, Yingxia Li and Lei Ma*
}

\begin{abstract}
Background: Gene fusion has epigenetic modification functions. The novel proteins encoded by gene fusion products play a role in cancer development. Therefore, a better understanding of the novel protein products may provide insights into the pathogenesis of tumors. However, the characteristics of chimeric genes are rarely studied. Here, we used weighted co-expression network analysis to investigate the biological roles and underlying mechanisms of chimeric genes.
\end{abstract}

Methods: Download the pig transcriptome data, we screened chimeric genes and parental genes from 688 sequences and 153 samples, predict their domains, and analyze their associations. We constructed a co-expression network of chimeric genes in pigs and conducted Gene Ontology enrichment and Kyoto Encyclopedia of Genes and Genomes pathway analysis on the generated modules using DAVID to identify key networks and modules related to chimeric genes.

Results: Our findings showed that most of the protein domains of chimeric genes were derived from fused pregenes. Chimeric genes were enriched in modules involved in the negative regulation of cell proliferation and protein localization to centrosomes. In addition, the chimeric genes were related to the growth factor- $\beta$ superfamily, which regulates cell growth and differentiation. Furthermore, in helper T cells, chimeric genes regulate the specific recognition of $\mathrm{T}$ cell receptors, implying that chimeric genes play a key role in the regulation pathway of T cells. Chimeric genes can produce new domains, and some chimeric genes are a key role involved in pathwayrelated function.

Conclusions: Most chimeric genes show binding activity. Domains of chimeric genes are derived from several combinations of parent genes. Chimeric genes play a key role in the regulation of several cellular pathways. Our findings may provide new directions to explore the roles of chimeric genes in tumors.

Keywords: Chimeric gene, Domain, WGCNA, Co-expression network, Tumor

\section{Introduction}

Chimeric genes are produced from the fusion of two or more parent genes [1] through chromosomal rearrangements, transcriptional read-through of adjacent genes, trans-splicing, and other mechanisms. Chimeric genes can be translated into new proteins with novel functions.

\footnotetext{
* Correspondence: mlei@shzu.edu.cn

College of Life Science, Shihezi University, Shihezi, Xinjiang 832000, China
Although proteins encoded by chimeric genes can show beneficial functions, the encoded proteins can also have deleterious functions. Chimeric genes are a cytogenetic feature of many cancers and have been used as diagnostic markers [2]. For example, the EML4 gene and the $A L K$ gene are fused into a chimeric gene [3], which has been used as a marker for advanced non-small cell lung cancer. Investigation into the protein domains encoded

C C The Author(s). 2021 Open Access This article is licensed under a Creative Commons Attribution 4.0 International License, which permits use, sharing, adaptation, distribution and reproduction in any medium or format, as long as you give appropriate credit to the original author(s) and the source, provide a link to the Creative Commons licence, and indicate if changes were made. The images or other third party material in this article are included in the article's Creative Commons licence, unless indicated otherwise in a credit line to the material. If material is not included in the article's Creative Commons licence and your intended use is not permitted by statutory regulation or exceeds the permitted use, you will need to obtain permission directly from the copyright holder. To view a copy of this licence, visit http://creativecommons.org/licenses/by/4.0/ The Creative Commons Public Domain Dedication waiver (http://creativecommons.org/publicdomain/zero/1.0/) applies to the data made available in this article, unless otherwise stated in a credit line to the data. 
by chimera may help provide insights into the cellular functions of the encoded proteins.

The features of the proteins produced by chimeric genes depend on the domains produced by parental genes. For example, in chronic myelogenous leukemia, the high tyrosine kinase activity of the chimeric $\mathrm{BCR}$ $\mathrm{ABL}$ protein is derived from the fusion of the phosphorylation domain encoded by the $B C R$ gene with the nonreceptor tyrosine kinase domain encoded from the $A B L$ proto-oncogene [4]. In early prostate cancer, the expression of erythroblast virus E26 carcinogen gene 2 (ERG) is increased through its fusion with the trans-membrane serine protease two gene (TMPRSS2) [5]. However, the principle that chimeric genes inherit domains from their parents requires further study. Normally, signal peptides (SP) direct chimeric proteins to their proper cellular and extracellular locations [6]. They are involved in the discovery of drug targets, protein production, and cancer biomarkers [7]. For example, the macrocyclic triamine cyclotriazadisulfonamide (CADA) decrease expression of specific proteins in a SP-dependent manner has opened the door to the possibility that the signal peptide becomes a validated target for drug design [8]. The signal peptide missense variant in cancer-brake gene CTLA4 was associated with lower risk and poor prognosis in breast carcinoma among Egyptian women, might have prognostic as well diagnostic impact in breast cancer [9]. Therefore, we took signal peptides as an example to explore the source of chimeric gene protein domains.

Gene design is a strategy to manufacture proteinencoding genes with specific biological functions. In these methods, gene sequences that encode different protein domains are fused to produce a fusion protein product with specific functions. For example, the artificially synthesized MGF-Ct24E peptide induces migration-promoting activity in human myogenic precursor cells and may be helpful for the treatment of Duchenne muscular dystrophy [10]. However, not all artificial fusion proteins perform the desired functions. For example, synthetic oligopeptides with selectin agglutination domains reduced ischemic damage at $24 \mathrm{~h}$ after transient focal cerebral ischemia, but did not reduce permanent focal cerebral ischemia [11]. Therefore, a better understanding of the characteristics of endogenous chimeric genes may be useful to guide gene design and synthesis.

Pigs have been used as large mammal models in various research studies [12]. Pigs are highly similar to humans not only in body weight, physiological characteristics, organ formation, and disease occurrence, but also in genomic sequence and chromosomal structure [13]. To explore the structural characteristics of chimeric genes in pigs, we used weighted co-expression network analysis (WGCNA) to investigate the role of chimeric genes in the network. Our results showed that the formation of a chimeric gene not only enrich the diversity and complexity of the transcription and protein, and provides a reference for the study of human chimeric genes.

\section{Materials and methods \\ Data preparation}

To define chimeric mRNA sets, mRNA datasets were downloaded from the Nucleotide database of the National Center for Biotechnology Information (NCBI, http://www. ncbi.nlm.nih.gov/nucleotide/, September 2016) [14], containing a total of 688 sequences (see Additional file 1). Pig reference genome sequence (Sus Sscrofa 10.2) was downloaded from the Ensembl Genome Browser (http://asia. ensembl.org/index.html, September 2016) [15]. Then, the mRNA reads were aligned to a pig reference genome sequence (Sus Sscrofa10.2). When a single mRNA sequence was aligned to multiple locations of the reference genome, only $0.5 \%$ of the homology level of the reference genome sequence was retained and at least $96 \%$ of the gene sequences were identical to the mRNA sequence. We obtained 1007 chimeric RNAs.

\section{Prediction of chimeric and parental protein domains}

We download the ncbi-blast-2.2.25 -x64-Win64 version to build a local BLAST (ftp://ftp.ncbi.nlm.nih.gov/blast/ executables/blast+/LATEST) [16] and compared 1007 chimeric mRNA with the mRNA dataset to predict the parent genes of the chimeric genes. Parameters were set as follows: (i) similarity (\% identity) $>95 \%$, (ii) left and right base alignment lengths $>90 \%$, and (iii) $E$ value of the comparison $<10^{-5}$ [17]. A total of 447 chimeric mRNAs matched to two parent genes.

We used Open Reading Frame Finder (ORF Finder, http://www.bioinformatics.org/sms2/orf_find.html) [18] from NCBI to predict the amino acid sequence of the chimeric mRNAs. The parameter settings were as follows: (i) minimal ORF length: $75 \mathrm{nt}$, (ii) ORF start codon: "ATG" only, (iii) genetic code: standard, (iv) amino acid length: greater than 100, and (v) the positive chain: retained.

SMART (http://smart.embl-heidelberg.de/) [19] and Universal Protein Resource (UniProt, http://www. uniprot.org/uniprot/?query=pyrin\&sort=score) [20] were used to predict the domains encoded by chimeric and parental genes. The parameter setting was as follows: remove the hidden| overlap domain. SignalP4.1 (http:// www.cbs.dtu.dk/services/SignalP/) was used to predict sequences encoding signal peptides in chimeric and parent genes [21].

Enrichment analysis of chimeric and non-chimeric genes The functional enrichment analysis of chimeric and nonchimeric genes was performed using the Database for 
Annotation, Visualization and Integrated Discovery (DAVID, https://david.ncifcrf.gov/) [22], and the false discovery rate (FDR) value less than 0.05 indicated significance. The pig genome level was used as the background for statistical analysis of enrichment.

\section{Construction of the gene co-expression network}

Download the pig transcript expression, including 153 samples (see Additional file 2): (i) if the number of genes expressing 0 in a sample accounts for more than $20 \%$ of the total number, the sample is deleted; (ii) genes with expression standard deviation greater than 5 were selected; and (iii) cluster samples and delete outliers.

We used the WGCNA package [23], dynamicTreeCut package [24], and FastCluster package [25] in R (version 4.02 ) to construct a co-expression network for pigs. The specific process is described in reference [26].

\section{Functional enrichment of module}

Functional enrichment analysis was performed using DAVID [27], using an FDR value of less than 0.05 to indicate significance. The pig genome level was used as the background for statistical analysis of enrichment.

\section{Pathway involved in chimeric genes}

We used DAVID for Kyoto Encyclopedia of Genes and Genomes (KEGG) path mining [27], using an FDR value less than 0.05 to indicate significance. The background of statistical analysis is based on the genome level of pigs.

\section{Results}

\section{Distribution of chimeric domains}

Domains of 1007 chimeric genes were predicted, and 1942 protein domains and 582 protein domain types were obtained. We analyzed the distribution frequency of the 582 protein domain types (excluding Signal peptides) and found that most domains only appeared once or twice. The results showed that only the 3\% (20/582) of chimeric domain occurrences number greater than 15, such as ZnF, coiled coil, WD40, EFh, LIM, HAT, and IG.

In order to obtain the domain indicators that can be used as fusion events, we compared the top 20 chimeric domains with the porcine genome domains and found that WD40, EFh, RRM, SH3, and SH2 were significantly enriched in the chimeric domains (Fisher's exact test, $p$ $<0.001$, Table 1). In addition, the overall distribution rate of chimeric protein domains is similar to the distribution of pig genome protein domains (Fisher's exact test, $p=0.3582$, Table 1 ).

\section{Signal peptides encoded by the chimeric}

To provide a real-world example on the origin of domains encoded by chimeric mRNAs, we used the signal peptide as an example. A signal peptide is a 5-30 amino acid peptide located in the $\mathrm{N}$-terminus of secretory proteins. Signal sequences have a tripartite structure, consisting of a hydrophobic core region (h-region) flanked by an $\mathrm{n}$-region and c-region. The latter region contains the signal peptidase consensus cleavage site.

As shown in Fig. 1, the chimera can obtain signal peptides through several mechanisms. (i) The signal peptides can be derived from the head parent (HP), (ii) the signal peptide can be derived from the tail parent (TP), regardless of whether the HP has the signal peptide; the HP became untranslated region (UTR) and the TP offered coding sequences, forming a $5^{\prime}$ UTR-coding sequence structure, and (iii) signal peptides can be re-built by connecting parent sequences. For example, an incomplete signal sequence of the HP obtained a cleavage site from the TP and (iv) reading-frame shift either creates or destroys signal peptide.

\section{Comparison of protein domains between chimeric and parental genes}

Among the 1007 chimeric genes, there are 447 two parent chimeric genes, 430 one parent chimeric genes, and 130 chimeric genes with no results. Analysis of each of 447 chimeric genes that matched two parent genes showed that although these chimeric genes contained domains of the parent genes, the chimeric genes were not just a combination of their two parent protein domains. Approximately 61\% (273/447) of these chimeric genes retained the domains of their parent genes. Among the 273 chimeric genes, 52 were identical with their parental genes, 94 retained the domain of one parent gene, and 127 contained domains of the two parent genes. The remaining 106 chimeric genes (24\%, 106/ 447) contained novel domains not found in parent genes. Approximately 15\% (68/447) of the chimeric gene does not contain the domain of their parent.

Table 1 Distribution characteristics of chimeric domains

\begin{tabular}{|c|c|c|c|c|c|c|c|c|c|c|c|}
\hline $\begin{array}{l}\text { Data } \\
\text { set }\end{array}$ & $\begin{array}{l}\text { Overall gene } \\
\text { number }\end{array}$ & $\begin{array}{l}\text { Gene number } \\
\text { (domains) }\end{array}$ & $\begin{array}{l}\text { Domain } \\
\text { type }\end{array}$ & $\begin{array}{l}\text { Overall domain } \\
\text { number }\end{array}$ & $\begin{array}{l}\text { WD40 } \\
\text { (\%) }\end{array}$ & $\begin{array}{l}\text { EFh } \\
(\%)\end{array}$ & $\begin{array}{l}\text { RRM } \\
\text { (\%) }\end{array}$ & $\begin{array}{l}\text { SH3 } \\
(\%)\end{array}$ & $\begin{array}{l}\text { LRR } \\
(\%)\end{array}$ & $\begin{array}{l}\mathrm{SH} 2 \\
(\%)\end{array}$ & $\begin{array}{l}\text { ANK } \\
(\%)\end{array}$ \\
\hline Chimeric & 1007 & 868 & 582 & 1942 & 8.9 & 5.3 & 4.0 & 3.3 & 2.9 & 2.7 & 2.7 \\
\hline Genome & 25882 & 23300 & 5943 & 59,701 & 2.9 & 0.7 & 0.7 & 0.5 & 2.2 & 0.3 & 2.3 \\
\hline P & & & $>0.05$ & & $<0.01$ & & & & & & \\
\hline
\end{tabular}

Note: $P$, the result of Fisher's exact test 


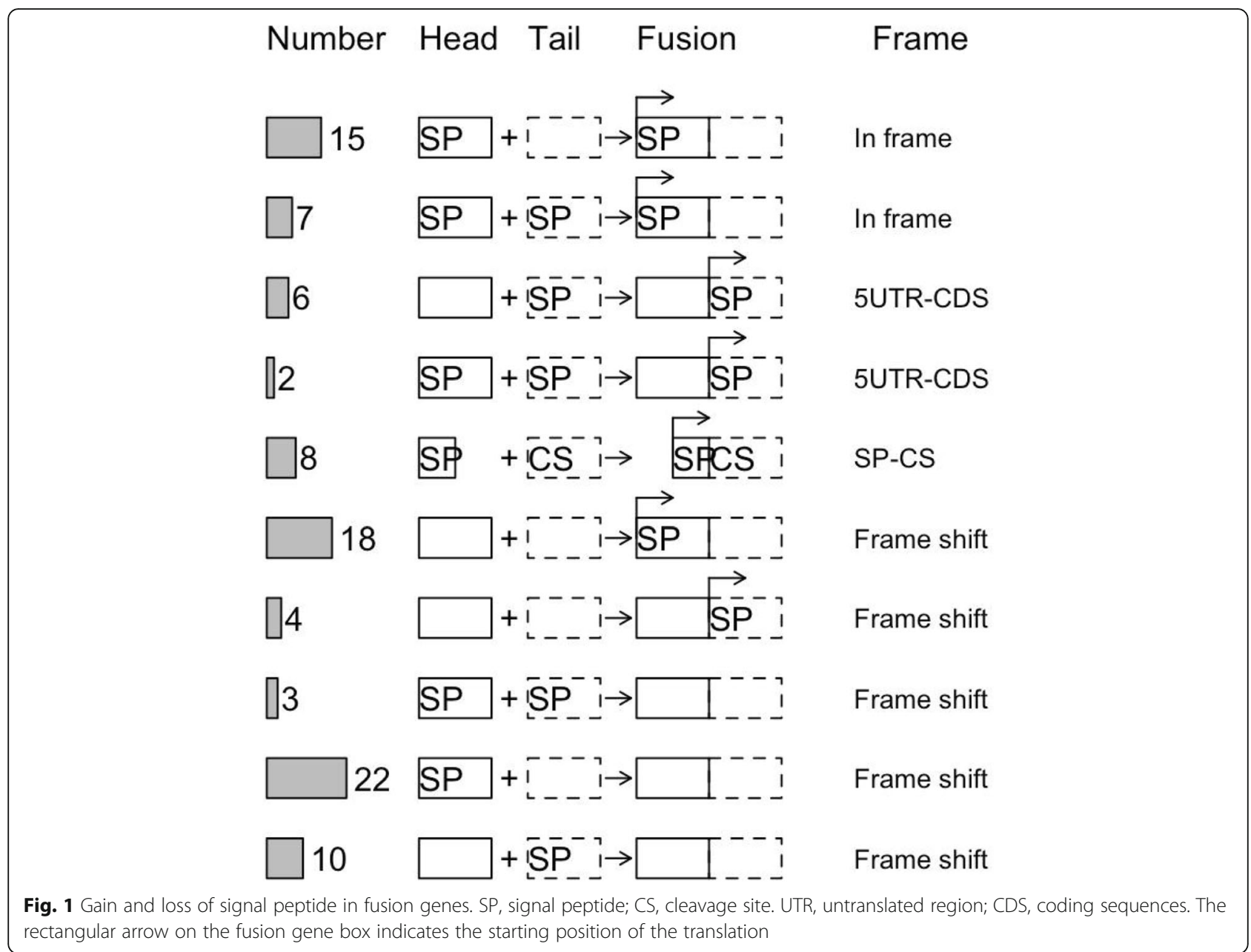

There were 338 domain types in the 447 chimeric genes, and their sources were analyzed statistically (Table 2). A total of 140 domain types were derived from only one parent gene. Among the 140 domain types, 60 types come from $5^{\prime}$ parent genes, 80 types come from 3' parent genes, 78 types come from both parent genes, 34 types resulted in a reading frame shift, and 86 types have no confirmed source.

\section{Construction of chimeric gene co-expression modules}

Using the abundance values of 475 chimeric genes and 2433 non-chimeric genes in 153 pig RNA-sequencing samples, we constructed 19 gene co-expression modules (Fig. 2; Table 3). The number of transcripts varied in the modules. The largest module, \#1, contained 479 transcripts while the smallest module \#19 contained only 32 transcripts. Furthermore, the number of chimeric transcripts also varied in the modules.

\section{Functional enrichment analysis}

Enrichment analysis using DAVID showed that the functions of chimeric genes were different compared with those of non-chimeric genes (FDR $<0.05$ ). For biological processes, chimeric genes were enriched in biologic

Table 2 Source of domains in chimeric genes

\begin{tabular}{|c|c|c|c|}
\hline Number of chimeric domains & 5 ' parent & 3' parent & Source \\
\hline 60 & + & - & 5' parent \\
\hline 80 & - & + & 3' parent \\
\hline 78 & + & + & $5^{\prime}$ parent and $3^{\prime}$ parent \\
\hline 34 & - & - & Reading frame shift \\
\hline 86 & $+/-$ & $+/-$ & $5^{\prime}$ parent or $3^{\prime}$ parent or reading frame shift \\
\hline
\end{tabular}

"+": the domain of the chimeric gene is derived from the parent gene. "-": the domain of the chimeric gene does not belong to the parent gene. "l": or 


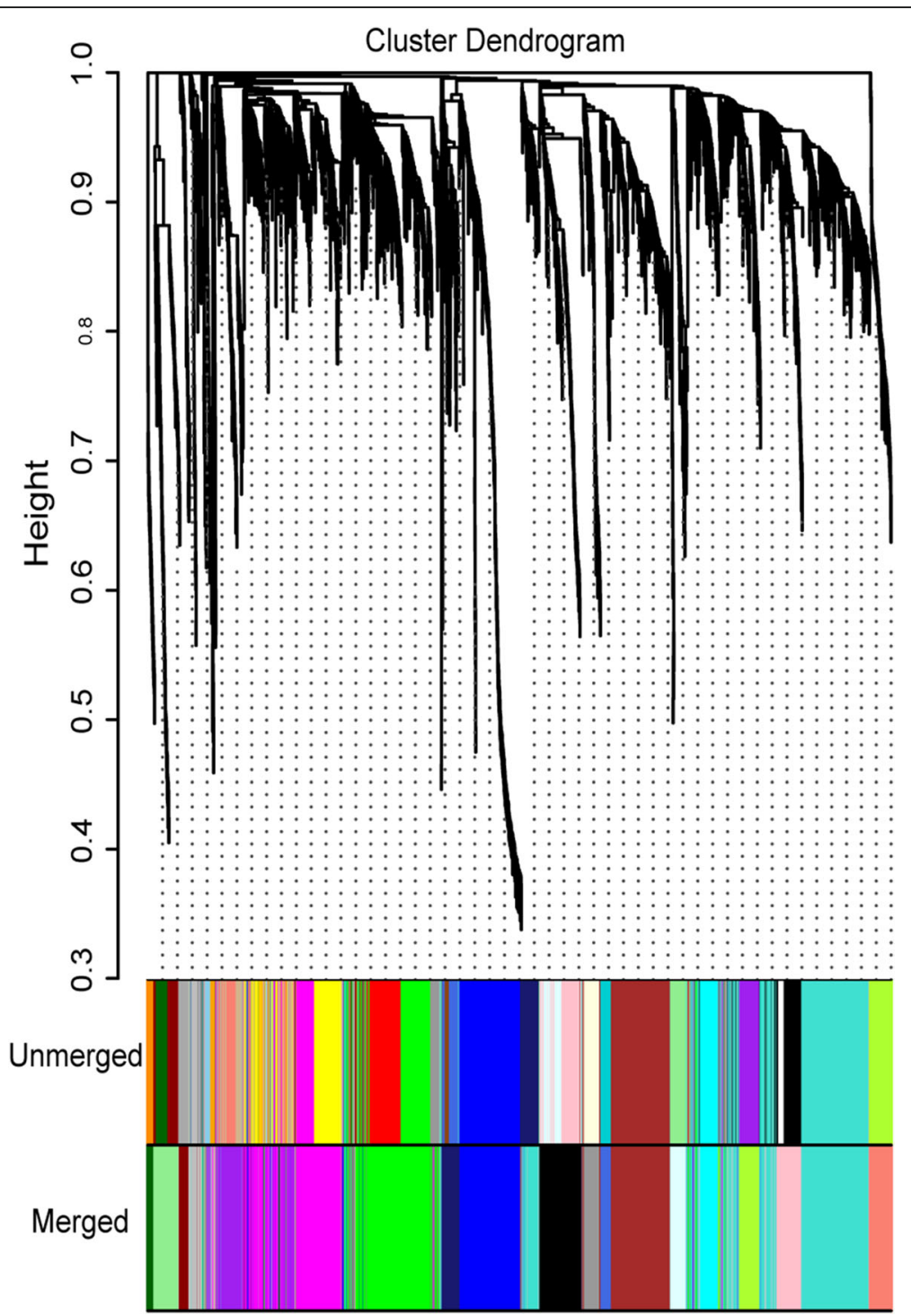

Fig. 2 The cluster of transcriptions and construction of modules. Different colors represent different modules. Cluster dendrogram, transcriptions cluster; unmerged, preliminary module construction; merged, integrated module

regulation and single organism process while nonchimeric genes were enriched in cellular and metabolic processes (Fig. 3a). For molecular functions, chimeric genes showed functions in binding while non-chimeric genes showed functions in catalytic activity (Fig. 3b). For the cytology component, chimeric genes are involved in cells while non-chimeric genes are involved in organelles (Fig. 3c).

\section{Functional enrichment analysis in specific modules}

The functional correlation of genes between modules was validated by enrichment analysis of the chimeric genes in modules \#1-5. The chimeric genes in different modules were enriched to the same function. Module $\# 1$, module $\# 3$, and module \#4 were enriched in the cytoplasm while module \#5 was enriched in the nucleus. Module \#2 was enriched in extracellular exosomes. However, in module $\# 1$, the chimeric genes were enriched in different functions (Fig. 4).

\section{Module visualization}

The relationship between chimeric and non-chimeric genes in the network was revealed by analyzing the coexpression of genes in modules \#4 and module \#5. As shown in Fig. 5, the chimeric genes appear more frequently than non-chimeric genes. This network is mainly related to the transforming growth factor- $\beta$ superfamily, which plays a role in regulating cell growth and differentiation. In this network, chimeric genes (AK461808, AK393675, AK233605, AK230955) and non-chimeric 
Table 3 Co-expression network module information containing chimeric genes

\begin{tabular}{|c|c|c|c|c|}
\hline$\#$ & Color in Fig. 2 & Cluster gene number & Number of chimeric genes & Chimeric proportion \\
\hline 1 & Turquoise & 479 & 80 & $16.70 \%$ \\
\hline 2 & Green & 367 & 60 & $16.30 \%$ \\
\hline 3 & Magenta & 324 & 55 & $17.00 \%$ \\
\hline 4 & Blue & 262 & 51 & $19.50 \%$ \\
\hline 5 & Brown & 250 & 35 & $14.00 \%$ \\
\hline 6 & Purple & 185 & 29 & $15.70 \%$ \\
\hline 7 & Black & 178 & 27 & $15.20 \%$ \\
\hline 8 & Pink & 126 & 13 & $10.30 \%$ \\
\hline 9 & Lightgreen & 103 & 14 & $13.60 \%$ \\
\hline 10 & Greenyellow & 83 & 13 & $15.70 \%$ \\
\hline 11 & Salmon & 83 & 17 & $20.50 \%$ \\
\hline 12 & Cyan & 82 & 11 & $13.40 \%$ \\
\hline 13 & Midnightblue & 72 & 13 & $18.10 \%$ \\
\hline 14 & Grey & 67 & 14 & $20.90 \%$ \\
\hline 15 & Grey60 & 62 & 10 & $16.10 \%$ \\
\hline 16 & Lightcyan & 61 & 12 & $19.70 \%$ \\
\hline 17 & Royalblue & 49 & 5 & $10.20 \%$ \\
\hline 18 & Darkred & 43 & 8 & $18.60 \%$ \\
\hline 19 & Darkgreen & 32 & 8 & $25.00 \%$ \\
\hline
\end{tabular}

Cluster gene number indicates the total gene number in each module, the number of chimeric genes indicates the chimeric gene number in each module, and the chimeric proportion indicates the number proportion chimeric genes in each module

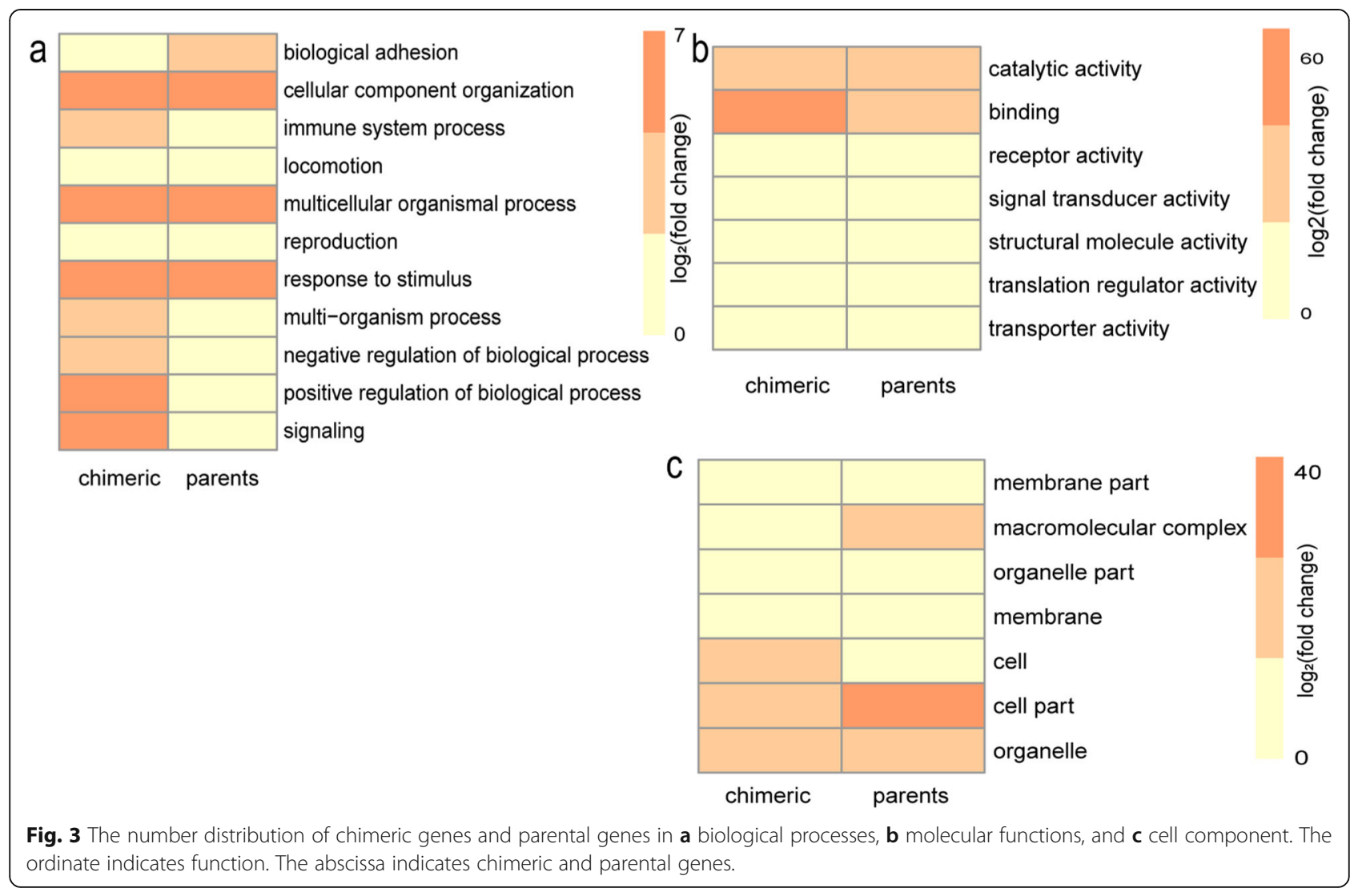




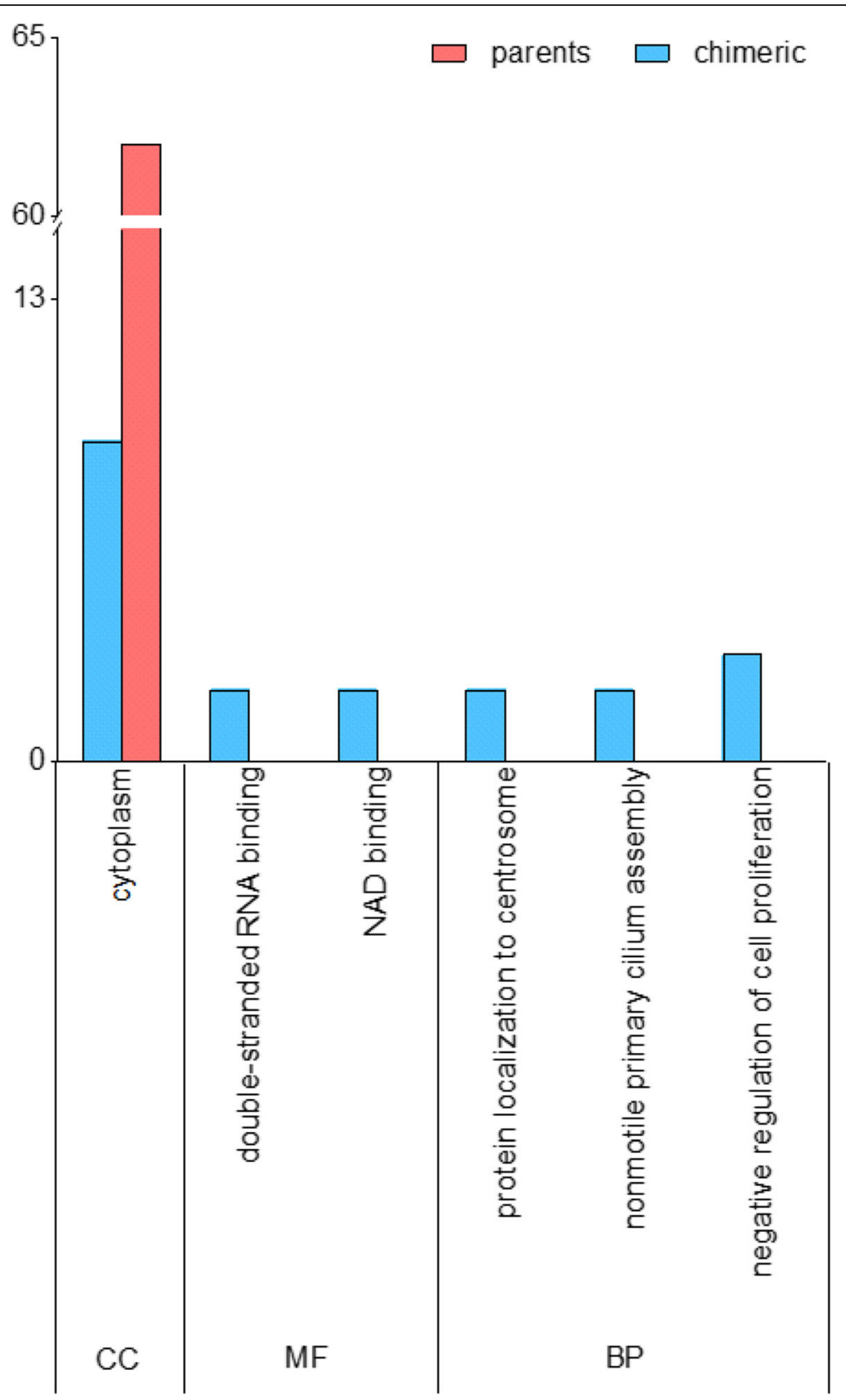

Fig. 4 The distribution of chimeric gene function enrichment in turquoise module. The abscissa indicates function. The ordinate indicates genes number. BP, biological processes; CC, cell component; MF, molecular function

genes (ENSSSCT00000010588, ENSSSCT00000007863) are connected to each other. They can regulate each other.

Chimeric genes are involved in the regulation of $\mathrm{T}$ cells We identified relationships between chimeric and nonchimeric mRNAs in various cellular pathways. As shown in Fig. 6, the chimeric gene (FJ944055) encodes the T cell antigen receptor (TCR) beta chain, which forms the TCR cell with the alpha chain. The chimera (FJ944055) can regulate the TCR to identify the antigen presented by the MHC molecule. Non-chimeric genes (AB602431, AK397194) are involved in the regulation of MHC class I (MHC-I) and MHC class II (MHC-II) molecules. MHC-I and MHC-II molecules bind to T cell antigen receptor (TCR) to activate CD8+ T cells and CD4+ T cells, respectively.

\section{Discussion}

The domains encoding by chimeric genes can be derived from parental genes in various ways. The domains and 


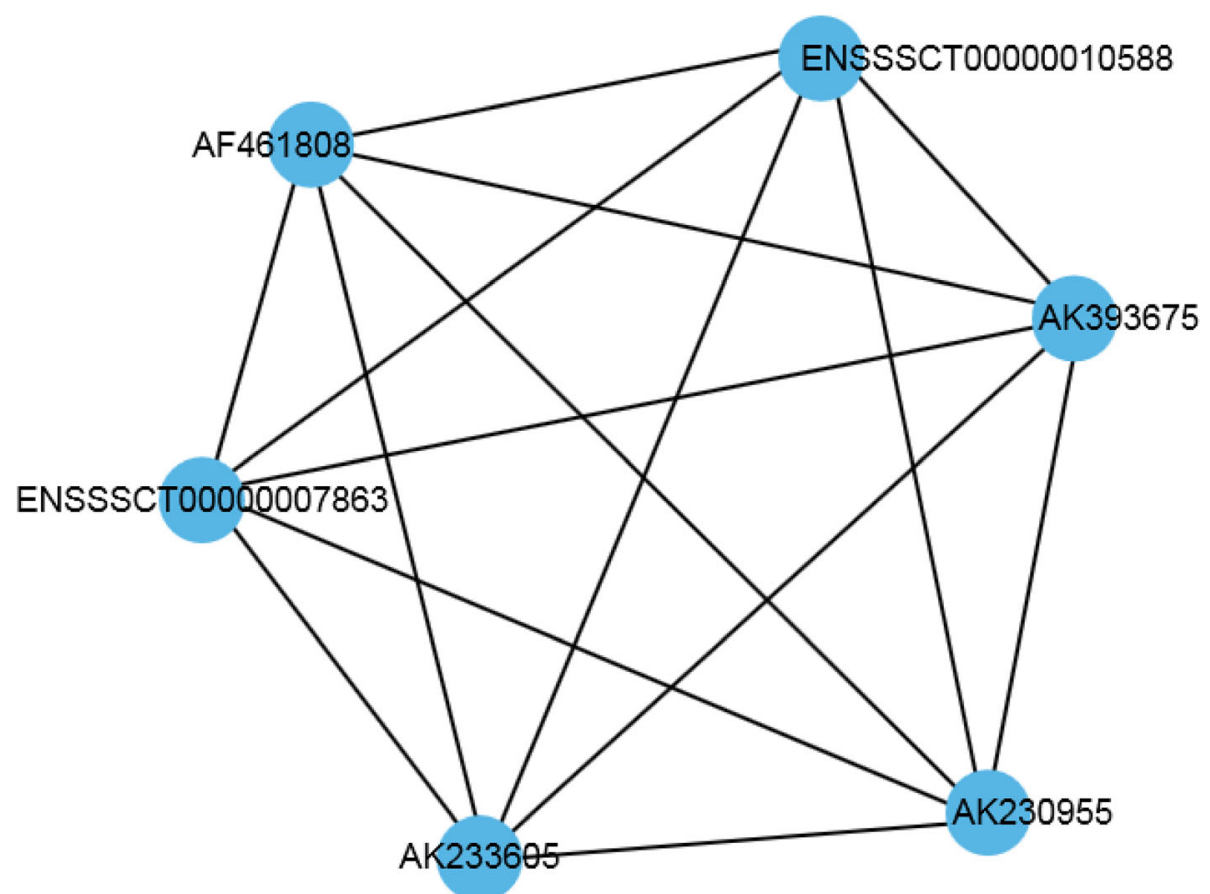

Fig. 5 The gene co-expression regulatory network of the third module and the fourth module. Line, a correlation between genes. The blue circle, gene registration number

functions of a chimeric gene may be the same as those in parent genes. For example, when genes that encode oncoproteins are fused, the chimeric genes may encode proteins that accelerate the division of cancer cells. However, most chimera encode both parental and novel domains. In cases in which a chimeric gene has a new function compared with the parent gene, it will suppress or promote the expression of the parent [28].

We used the signal peptide as an example to provide a real-world example on the origin of domains encoded by chimeric genes. A signal peptide is composed of about 5-30 amino acids and guides the transport of proteins

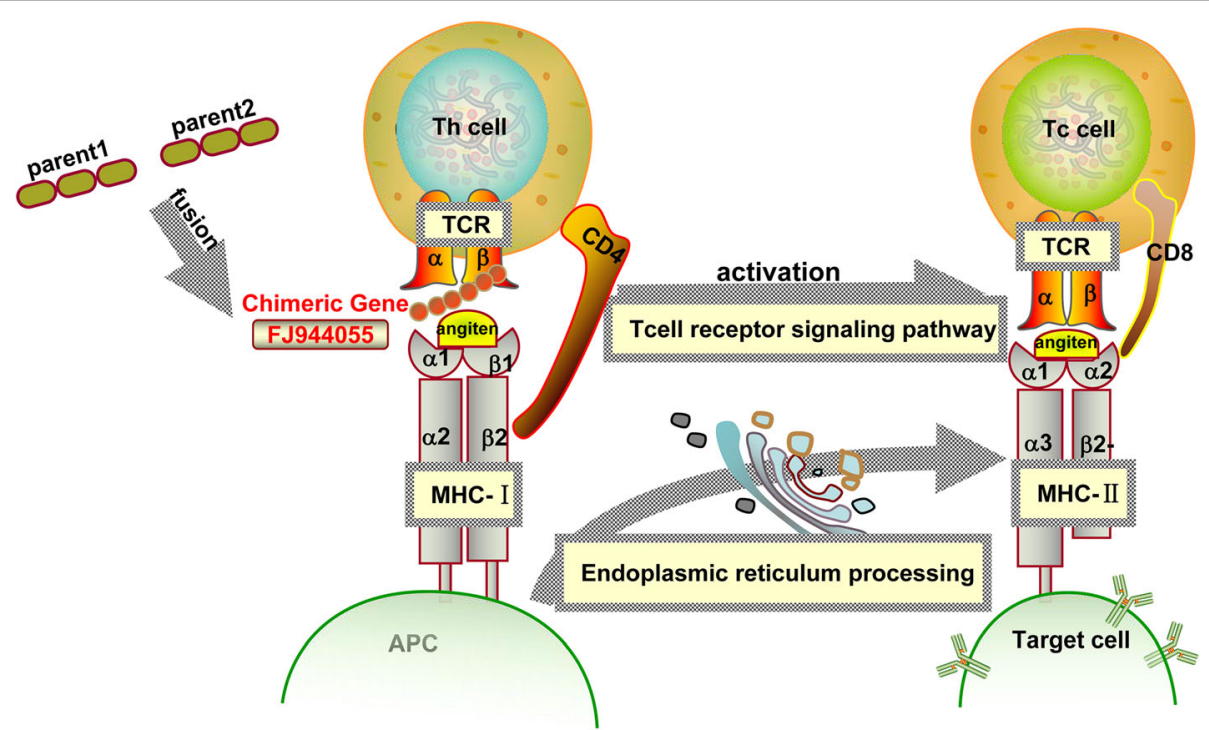

Fig. 6 The regulatory pathway of T lymphocytes. Th cell, helper T cell; Tc cell, cytotoxic T cell, TCR, T cell antigen receptor; APC, antigen-presenting cells 
through the cell membrane [6]. Signal peptides play different roles in chimeric genes. The LPCAT2-TXNDC5 chimeric product is derived from fusion of the LPCAT2 gene, which contains a signal peptide-encoding sequence, with the TXNDC5 gene, which lacks this sequence [29]. LPCAT2-TXNDC5 chimera is detected extracellular space, possibly from the protein being transported through the membrane. The gene that does not encode protein products can fuse with other genes, resulting in a fusion gene with protein-encoding capability. This may be due to signal peptides provided by other genes or proteins produced by a reading frameshift.

WGCNA can be used to find modules of highly related transcripts, help screen hub transcripts and identify candidate biomarkers [30]. Using WGCNA, we found that genes in the same module are functionally related to each other [31]. Chimeric genes and parent genes in the same module can simultaneously edit hexokinase. This result is consistent with the study showing that the $M Y B-Q K I$ chimeric gene regulates the same pathway as the parental gene [28]. The results also revealed specific regulatory relationships between chimeric genes and non-parent genes in different modules. KEGG pathway enrichment analysis revealed that the TCR-beta gene and pig MHC-I and MHC-II transcripts were enriched in viral myocarditis pathways. TCR identifies heterologous antigen through signal regulation, killer $\mathrm{T}$ cells identify MHC class I antigen, and helper T cells identify MHC class II antigen. In addition, TCR play functions in cancer, and TCR expression predicts prognosis for nonsmall cell lung cancer patients after curative surgery [32]. We hypothesized that TCR and MHC antigens recognized by TCR may exist and function in non-small cell lung cancer tissues. More studies are required to explore this possibility. Together these findings suggest regulatory relations between chimeric genes and parental genes in the same module and show that chimeric genes and non-chimeric genes have similar effects in different modules.

The studies on chimeric genes have mainly focused on a specific chimeric gene and explore the relation between its function and cancer occurrence. Our current study provides insights into the general characteristics of chimeric genes and systematically analyzes the role of chimeric genes in co-expression networks. For example, a previous study examined that the FOXO1-PAX3 chimeric genes as a focus of Alveolar rhabdomyosarcoma (ARMS), exploring its related regulatory network [33]. In the research, we integrated and compared pig transcriptional data with DNA data and identified 1007 chimeric genes. We used these chimeric genes to build a chimeric genes co-expression network using WGCNA. The results revealed a regulatory relationship between chimeric genes and non-chimeric genes. The specific regulatory networks between chimeric genes and nonchimeric genes require further study.

\section{Conclusions}

In conclusion, most chimeric genes show binding activity, and domains of the chimeric genes are derived from several combinations of parent genes. WD40, EFh, RRM, $\mathrm{SH} 3$, and $\mathrm{SH} 2$ domains may be used as domain indicators for fusion events. In our analyses, we detected differences in the number of chimeric genes in the modules. Chimeric genes play a key role in the regulation of several cellular pathways. These findings may provide new directions to explore the roles of chimeric genes in tumors.

\section{Abbreviations}

GO: Gene Ontology; KEGG: Kyoto Encyclopedia of Genes and Genomes; WGCNA: Weighted gene co-expression network analysis; DAVID: Database for Annotation, Visualization and Integrated Discovery; FDR: False discovery rate; HP: Head parent; TP: Tail parent; UTR: Untranslated region; CDS: Coding sequences; CS: Cleavage site; TCR: T cell antigen receptor

\section{Supplementary Information}

The online version contains supplementary material available at https://doi. org/10.1186/s12957-021-02248-9.

Additional file 1. (XLS $80 \mathrm{~kb}$ )

Additional file 2. (XLS $32 \mathrm{~kb}$ )

\section{Acknowledgements}

We thank all of the contributors of the RNA-seq data sets and the anonymous reviewers for helpful suggestions on the manuscript.

\section{Authors' contributions}

LM designed the study. PL and YL performed the data analyses. PL drafted the manuscript. LM revised the manuscript. The authors read and approved the final manuscript.

\section{Funding}

The research was supported by the National Natural Science Foundation of China (31860308, 31760302 and 31272416) and the Science Foundation of Shihezi University (RCZK201953). The funders had no role in the study design, data collection and analysis, decision to publish, or preparation of the manuscript.

Availability of data and materials

All data in this study were obtained from public databases.

\section{Declarations}

Ethics approval and consent to participate Not applicable.

Consent for publication

Not applicable.

Competing interests

The authors declare that they have no competing interests. 
Received: 20 December 2020 Accepted: 19 April 2021

Published online: 12 May 2021

\section{References}

1. Zhuo JS, Jing XY, Du X, Yang XQ. Generation of Chimeric RNAs by cissplicing of adjacent genes (cis-SAGe) in mammals. Yi Chuan. 2018;40(2):14554. https://doi.org/10.16288/j.yczz.17-197.

2. Wu H, Li X, Li H. Gene fusions and chimeric RNAs, and their implications in cancer. Genes Dis. 2019;6(4):385-90. https://doi.org/10.1 016/j.gendis.2019.08.002.

3. Wong DW, Leung EL, So KK, Tam IY, Sihoe AD, Cheng LC, et al. The EML4ALK fusion gene is involved in various histologic types of lung cancers from nonsmokers with wild-type EGFR and KRAS. Cancer-Am Cancer Soc. 2009; 115:1723-33.

4. Sharda S, Sarmandal P, Cherukommu S, Dindhoria K, Yadav M, Bandaru S, et al. A Virtual Screening Approach for the Identification of High Affinity Small Molecules Targeting BCR-ABL1 Inhibitors for the Treatment of Chronic Myeloid Leukemia. Curr Top Med Chem. 2017;17(26):2989-96. https://doi. org/10.2174/1568026617666170821124512.

5. Tomlins SA, Laxman B, Varambally S, Cao X, Yu J, Helgeson BE, et al. Role of the TMPRSS2-ERG gene fusion in prostate cancer. Neoplasia. 2008;10(2):17788. https://doi.org/10.1593/neo.07822.

6. Jiang Z, Niu T, LV X, Liu Y, Li J, Lu W, et al. Secretory expression fine-tuning and directed evolution of diacetylchitobiose deacetylase by Bacillus subtilis. Appl Environ Microbiol. 2019;85(17). https://doi.org/10.1128/AEM.01076-19.

7. Lai JS, Cheng CW, Sung TY, Hsu WL. Computational comparative study of tuberculosis proteomes using a model learned from signal peptide structures. Plos One. 2012;7(4):e35018. https://doi.org/10.1371/journal.pone. 0035018.

8. Lumangtad LA, Bell TW. The signal peptide as a new target for drug design. Bioorg Med Chem Lett. 2020;30(10):127115. https://doi.org/10.1016/j.bmcl.2 020.127115 .

9. Babteen NA, Fawzy MS, Alelwani W, Alharbi RA, Alruwetei AM, Toraih EA, et al. Signal peptide missense variant in cancer-brake gene CTLA4 and breast cancer outcomes. Gene. 2020;737:144435. https://doi.org/10.1016/j. gene.2020.144435.

10. Mills P, Lafreniere JF, Benabdallah BF, El FEM, Tremblay JP. A new promigratory activity on human myogenic precursor cells for a synthetic peptide within the $\mathrm{E}$ domain of the mechano growth factor. Exp Cell Res. 2007;313(3):527-37. https://doi.org/10.1016/j.yexcr.2006.10.032.

11. Kaur P, Liu F, Tan JR, Lim KY, Sepramaniam S, Karolina DS, et al. Non-coding RNAs as potential neuroprotectants against ischemic brain injury. Brain Sci. 2013;3(4):360-95. https://doi.org/10.3390/brainsci3010360.

12. Huckenpahler AL, Carroll J, Salmon AE, Sajdak BS, Mastey RR, Allen KP, et al. Noninvasive imaging and correlative histology of cone photoreceptor structure in the pig retina. Transl Vis Sci Technol. 2019;8(6):38. https://doi. org/10.1167/tvst.8.6.38.

13. Umu OC, Frank JA, Fangel JU, Oostindjer M, Da SC, Bolhuis EJ, et al. Resistant starch diet induces change in the swine microbiome and a predominance of beneficial bacterial populations. Microbiome. 2015;3(1):16. https://doi.org/10.1186/s40168-015-0078-5.

14. Kodama $Y$, Shumway M, Leinonen R. The Sequence Read Archive: explosive growth of sequencing data. Nucleic Acids Res. 2012;40(D1):D54-6. https:// doi.org/10.1093/nar/gkr854.

15. Kinsella RJ, Kahari A, Haider S, Zamora J, Proctor G, Spudich G, et al. Ensembl BioMarts: a hub for data retrieval across taxonomic space. Database (Oxford). 2011;2011:r30.

16. Camacho C, Coulouris G, Avagyan V, Ma N, Papadopoulos J, Bealer K, et al. BLAST+: architecture and applications. BMC Bioinformatics. 2009;10(1):421. https://doi.org/10.1186/1471-2105-10-421.

17. Ma L, Yang S, Zhao W, Tang Z, Zhang T, Li K. Identification and analysis of pig chimeric mRNAs using RNA sequencing data. BMC Genomics. 2012; 13(1):429. https://doi.org/10.1186/1471-2164-13-429.

18. Xu TP, Ma P, Wang WY, Shuai Y, Wang YF, Yu T, et al. KLF5 and MYC modulated LINC00346 contributes to gastric cancer progression through acting as a competing endogeous RNA and indicates poor outcome. Cell Death Differ. 2019;26(11):2179-93. https://doi.org/10.1038/s41418-01 8-0236-y.

19. Gould CM, Diella F, Via A, Puntervoll P, Gemund C, Chabanis-Davidson S, et al. ELM: the status of the 2010 eukaryotic linear motif resource. Nucleic Acids Res. 2010;38(suppl_1):D167-80. https://doi.org/10.1093/nar/gkp1016.
20. Apweiler R, Bairoch A, Wu CH, Barker WC, Boeckmann B, Ferro S, et al. UniProt: the Universal Protein knowledgebase. Nucleic Acids Res. 2004; 32(90001):D115-9. https://doi.org/10.1093/nar/gkh131.

21. Emanuelsson O, Brunak S, von Heijne G, Nielsen $\mathrm{H}$. Locating proteins in the cell using TargetP, SignalP and related tools. Nat Protoc. 2007;2(4):953-71. https://doi.org/10.1038/nprot.2007.131.

22. Jiao X, Sherman BT, Huang DW, Stephens R, Baseler MW, Lane HC, et al. DAVID-WS: a stateful web service to facilitate gene/protein list analysis. Bioinformatics. 2012;28(13):1805-6. https://doi.org/10.1093/bioinformatics/ bts251.

23. Di Y, Chen D, Yu W, Yan L. Bladder cancer stage-associated hub genes revealed by WGCNA co-expression network analysis. Hereditas. 2019;156(1): 7. https://doi.org/10.1186/s41065-019-0083-y.

24. Ma J, Li R, Wang J. Characterization of a prognostic fourgene methylation signature associated with radiotherapy for head and neck squamous cell carcinoma. Mol Med Rep. 2019;20:622-32.

25. Moraru C, Varsani A, Kropinski AM. VIRIDIC-A Novel Tool to Calculate the Intergenomic Similarities of Prokaryote-Infecting Viruses. Viruses. 2020;12(11). https://doi.org/10.3390/v12111268.

26. Langfelder P, Horvath S. WGCNA: an R package for weighted correlation network analysis. BMC Bioinformatics. 2008;9(1):559. https://doi.org/10.11 86/1471-2105-9-559.

27. Dennis GJ, Sherman BT, Hosack DA, Yang J, Gao W, Lane HC, et al. DAVID: Database for Annotation, Visualization, and Integrated Discovery. Genome Biol. 2003;4(5):P3. https://doi.org/10.1186/gb-2003-4-5-p3.

28. Bandopadhayay P, Ramkissoon LA, Jain P, Bergthold G, Wala J, Zeid R, et al. MYB-QKI rearrangements in angiocentric glioma drive tumorigenicity through a tripartite mechanism. Nat Genet. 2016;48(3):273-82. https://doi. org/10.1038/ng.3500.

29. Zhang L, Hou Y, Li N, Wu K, Zhai J. The influence of TXNDC5 gene on gastric cancer cell. J Cancer Res Clin Oncol. 2010;136(10):1497-505. https:// doi.org/10.1007/s00432-010-0807-x.

30. Yepes S, Lopez R, Andrade RE, Rodriguez-Urrego PA, Lopez-Kleine L, Mercedes TM. Co-expressed miRNAs in gastric adenocarcinoma. Genomics. 2016;108(2):93-101. https://doi.org/10.1016/j.ygeno.2016.07.002.

31. Wan Q, Tang J, Han Y, Wang D. Co-expression modules construction by WGCNA and identify potential prognostic markers of uveal melanoma. Exp Eye Res. 2018;166:13-20. https://doi.org/10.1016/j.exer.2017.10.007.

32. Song $Z$, Chen $X$, Shi $Y$, Huang $R$, Wang W, Zhu K, et al. Evaluating the potential of $T$ cell receptor repertoires in predicting the prognosis of resectable non-small cell lung cancers. Mol Ther-Meth Clin D. 2020;18:73-83. https://doi.org/10.1016/j.omtm.2020.05.020.

33. Thanh HN, Barr FG. Therapeutic approaches targeting PAX3-FOXO1 and its regulatory and transcriptional pathways in rhabdomyosarcoma. Molecules. 2018;23. https://doi.org/10.3390/molecules23112798.

\section{Publisher's Note}

Springer Nature remains neutral with regard to jurisdictional claims in published maps and institutional affiliations.

Ready to submit your research? Choose BMC and benefit from:

- fast, convenient online submission

- thorough peer review by experienced researchers in your field

- rapid publication on acceptance

- support for research data, including large and complex data types

- gold Open Access which fosters wider collaboration and increased citations

- maximum visibility for your research: over $100 \mathrm{M}$ website views per year

At BMC, research is always in progress.

Learn more biomedcentral.com/submissions 